\title{
Developing a Clinical Practice Guideline for Surgical Diabetic Patients
}

\author{
Barbie Harbaugh, DNP, MSN, RN \\ Walden University, Minneapolis, Minnesota, United States \\ Diane Whitehead, EdD, DNP, RN, ANEF \\ Walden University, Minneapolis, Minnesota, United States
}

Contact: $\underline{\text { bhoover@pct.edu }}$

\begin{abstract}
Diabetes is a recognized risk factor for postoperative infection, acute renal failure, ileus, and a lengthy hospital stay. Optimal screening, management, and scheduling of elective surgery for diabetic patients have been shown to improve quality care, decrease complications, increase the efficiency, and lower the costs of preoperative patient care. However, surgery cancellations are common due to inadequate preoperative glycemic control and poor intraoperative glycemic control, which are recognized risk factors for perioperative or postoperative complications. There were no clinical practice guidelines or optimization protocols for elective surgery patients at a small rural hospital in the northeast United States. The purpose of this project was to develop a clinical practice guideline for elective surgery patients in this hospital outlining the acceptable HgbA1C level for surgical clearance. The five attributes of change, individual and collective leadership, operational support, fostering relationships, organizational learning, and balance, framed the development of this project. Based on the current evidence, the $\mathrm{HgbA1C}$ level approved to be acceptable for surgery clearance was $8.5 \% \mathrm{mg} / \mathrm{dL}$. An 18 -member expert panel consisting of anesthesiologists, nurse anesthetists, an endocrinologist, a diabetic nurse educator, an administrator, physician assistants, nurse practitioners, and surgeons reviewed the proposed guideline using the AGREE II tool. Using a scale of 1 to 7 (strongly disagree to strongly agree), the team members agreed with the proposed guideline, with a score of 6 or higher in each domain. Utilization of this guideline may promote positive social change by addressing the gap in practice at this hospital and significantly reducing the number of surgery cancellations among diabetic patients.
\end{abstract}

Keywords: clinical practice guideline; preoperative optimization; diabetes optimization; elective surgery; HgbA1C; glycosylated hemoglobin; A1C, surgery optimization; elective surgery diabetic patients

Date Submitted: September 13, 2020 | Date Published: October 20, 2020

Recommended Citation

Harbaugh, B., \& Whitehead, D. (2020). Developing a clinical practice guideline for surgical diabetic patients. Journal of Excellence in Nursing and Healthcare Practice, 2, 24-30. https://doi.org/10.5590/JENHP.2020.2.1.03

\section{Introduction}

Diabetes is a recognized risk factor for postoperative infection, acute renal failure, ileus, and a lengthy hospital stay. Poor preoperative glycemic control portends poor intraoperative glycemic control, which is an 
established risk factor for perioperative morbidity (Turner et al., 2018). Surgical patients with perioperative hyperglycemia have a higher risk for infection and associated adverse consequences after surgery compared to patients without hyperglycemia. When patients with poorly controlled diabetes present for surgery, they impose a significant financial health resource burden, including prolonged ventilator dependence, longer hospital stay, and greater postoperative loss of productivity (Turner et al.). As the incidence of diabetes increases, optimal screening, management, and scheduling of elective surgery for patients with diabetes have become issues of increasing significance. Although analysis of the cost-effectiveness of postponing scheduled surgery to treat poor glycemic control in pre-surgical populations is crucial for enhancing the value proposition of the pronouncement to have surgery, the optimal preoperative care delivery model for diabetes management remains unclear (Turner et al.). Hospitals have been continually exploring methods to reduce operational costs while providing safe, efficient delivery of healthcare in a changing healthcare system.

Implementation of the Affordable Health Care Act in 2010 for healthcare reform has been one of the major driving forces to reduce costs in the health care system. Operating rooms have been one of the costliest areas of hospital operations, and, with growing concerns to lower health care costs, hospitals have been faced with multiple mounting financial pressures.

Surgical operating rooms are vital resources for patient care and financial profitability and are often the largest contributors to a hospital's financial success. Surgical cancellations can negatively impact an organization's financial revenue; therefore, efficient utilization of operating room time is critical to reducing expenses (Minor, 2018).

\section{Framework}

A concept analysis on change completed by Nelson-Brantley and Ford (2017) identified five attributes important to successful change initiative. These five attributes, individual and collective leadership, operational support, fostering relationships, organizational learning, and balance, framed the development of this project.

Individual and collective leadership. The anesthesiologists and nurse anesthetists at this small rural hospital identified the problem of same-day cancellations. They were experiencing patient dissatisfaction when their surgery was being cancelled upon arrival to the department in the morning. The anesthesia department was also experiencing the discontent of the surgeon groups because they were suddenly with an open surgery slot. However, when the issue was brought to the table, all levels of management, department heads, collaborating departments, and staff were ready to problem-solve and seek solutions.

Operational support. Collective leadership also meant that the project had full operational support, including manpower, budgetary funds, engineering resources, and quality improvement monitoring.

Fostering relationships. The project began with perceptive inquiry. We conducted interviews with staff, surgeons, anesthesia staff, administrative personnel, and patients to seek to understand the issue of lastminute surgery cancellations. We spent hours in observation in various departments to discern the patient flow through the preadmission process. Time was spent analyzing the preadmission testing process from start to finish and the anesthesia policies and protocols, which were compared to the evidence in the literature. During all these explorations, we used open-ended inquiry from a non-biased approach. This approach assisted in the fostering of relationships as the project progressed through its stages.

Organizational learning. Organizational learning was defined as the process of change in thought and action, embedded in and affected by the institutions of the organization. As we searched the evidence in the literature concerning diabetes optimization, documented clinical practice guidelines, and the impact of 
Harbaugh \& Whitehead, 2020

surgery cancellations on the hospital system, information sharing took place consistently and constantly. Evidence in the literature gave support towards developing a facility-specific clinical practice guideline. Evidence in the professional databases validated the recommended $\mathrm{HgbA1C}$ level acceptable for preoperative surgery consideration. Evidence in the literature revealed fiscal implications for the facility when surgeries are cancelled at the last minute. Finally, substantiation in the literature validated patient concerns and dissatisfaction with the realization of a surgery being cancelled even after progressing through the preadmission certification process.

Balance. Nurses leading change must balance creating a sense of urgency with realistic patience, toughness with empathy, optimism with realism, and self-reliance with trust in others. To effectively lead change, nurses must balance their ability to be caring and supportive with showing more proactive behavior in ensuring their voice is heard at the table of change efforts (Bunker, 2006).

\section{Literature Review}

Diabetes is a substantial risk element for problems following many types of surgery. Diabetes increases the incidence of infection, as well as general morbidity and mortality, and is associated with other comorbidities, which increase the risk of surgical intervention. Additionally, perioperative short-term glycemic control is associated with poor surgical outcomes both in patients with and without diabetes, underpinning the role of stress hyperglycemia in this relationship (Rollins et al., 2016).

Glycosylated hemoglobin (HgbA1C) has been used as a measure of diabetic control, reflecting long-term glucose concentrations over the preceding months, and tight control is associated with reduced incidence and slower progression of diabetes-related complications, myocardial infarction, and stroke (Rollins et al., 2016). The American Diabetes Association (ADA) (2019) released guidelines recommending that target HgbA1C for people with diabetes should be $<7 \%$.

Many surgeons and anesthesiologists seek glucose levels $<200 \mathrm{mg} / \mathrm{dL}$ on the day of surgery, although evidence in the literature suggests that primary care physicians have never ordered baseline HgbA1C on their diabetic patients preoperatively (LaBoone et al., 2014; Lee et al., 2014). Acute hyperglycemia during the perioperative period has been studied extensively in the literature. It is also connected to poor clinical outcomes in patients with and without diabetes. This connection is well recognized in the literature for hyperglycemia on the day of surgery, within 24-48 hours of surgery, and during the full hospital stay. Further, insulin infusion protocols designed to prevent hyperglycemia in the perioperative and postoperative period demonstrate improved surgical outcomes. However, few studies have examined the relationship between preoperative $\mathrm{HgbA1C}$ levels and surgical outcomes. Studies in the literature concerning patients undergoing surgery document an association between elevated $\mathrm{HgbA1C}$ values and surgical complications, including mortality, cerebrovascular accidents, and wound infection. Persistent chronic hyperglycemia (elevated $\mathrm{HgbA1C}$ ) is undoubtedly a predictor of long-standing complications of diabetes and is the key target for glycemic control in diabetes. It remains unclear whether chronic hyperglycemia has an adverse effect on surgical outcomes over and above acute perioperative hyperglycemia and whether standards of care that address elevated $\mathrm{HgbA1C}$ levels prior to surgery would improve clinical outcomes (Underwood et al., 2014). The ADA has consistently published guidelines for perioperative glycemic control, but there are no specific guidelines for preoperative $\mathrm{HgbA1C}$ recommendation for diabetes optimization preoperatively for the elective surgery patient. Because of the deficiency in data, arbitrary HgbA1C cutoffs are used by surgeons, anesthesiologists, internists, and endocrinologists. This rural hospital practiced in this manner.

The American Association of Diabetes Educators (Mendez et al., 2018) has recommendations for the glucose management of the diabetic patient preoperatively, perioperatively, and postoperatively with insulin types, sliding-scale management, and discharge insulin management. This professional organization does not 
address preoperative $\mathrm{HgbA1C}$ recommendations for the diabetic elective surgery patient. The following organizations specific to this project have no preoperative guidelines for optimal HgbA1C levels for the elective diabetic surgical patient: American Surgical Association, American College of Surgeons, American Society of Anesthesiologists, Anesthesia Patient Safety Foundation, American Association of Nurse Anesthetists, and the American Association of Peri-Anesthesia Nurses. These organizations do have some blood sugar guidelines for the perioperative phase of surgery; however, these guidelines do not apply to this project focus.

\section{Background}

A struggle to improve operating room efficiency was a significant priority, as health care costs became more challenging in a rural 160-bed acute care hospital in the northeast United States. Given that the epidemiological data suggest that "good" preoperative glycemic control is linked with a lower risk of postoperative complications, it has been promoted that $\mathrm{HgbA1C}$ concentrations should be optimized before an elective procedure (Levy \& Dhatariya, 2019). Stakeholders for this project included the pre-surgical diabetic patients, pre- and postoperative nursing staff, surgeons, hospital administration, and caregivers. Positive social change may occur for the patients, families, caregivers, and health care providers by improving the diabetic patients' quality of life and the financial outcomes for the facility. Based on current evidence, hospital administration proposed that implementing a diabetic optimization protocol to measure if a patient's health status is optimal during the preoperative consultation period could reduce operating room cancellations for "change in patient's medical condition" within 48 hours of the surgery date.

In an attempt to decrease the number of unnecessary elective surgery cancellations related to poor diabetes surgical optimization, practitioners in a small rural hospital formed a group consisting of anesthesiologists, nurse anesthetists, surgeons, administrators, endocrinologists, a diabetic nurse educator, same-day surgery staff, and preadmission testing staff, to establish a clinical practice guideline (CPG) for diabetes optimization. There was no CPG in place at this facility for consistent rulings on acceptable $\mathrm{HgbA1C}$ levels for elective sameday surgery patients. Given the epidemic levels of diabetes in the overall population, hyperglycemia around the time of surgery is often identified, with estimated rates of $80 \%$ in cardiac and $40 \%$ in non-cardiac surgical patients (Levy \& Dhatariya, 2019). The goal of this project was to review current evidence and guidelines and to develop a CPG that could be recommended to the surgical department in a small rural hospital. The practice question was: based on current evidence, what preoperative diabetic optimization protocol/CPG for adult elective surgery diabetic patients should be recommended for a small rural hospital?

\section{Clinical Practice Guideline}

To address the issue of canceled 1-day surgery cases related to poorly optimized diabetic patients, we developed a CPG to standardize the HgbA1C levels that would be accepted in the preadmission process to proceed with a scheduled surgery. A standardized timeframe for the completion of this HgbA1C test was set for the diabetic patient. Guidelines for the preadmission staff to follow for initiating diabetes optimization by an endocrinologist or the primary care physician when the $\mathrm{HgbA1C}$ level was $>8.5 \%$ were developed. Finally, after the optimization had been completed and laboratory tests had met the acceptable level, a protocol was written to reschedule the elective one-day surgery procedure.

The expert panel was initiated to review the initial CPG proposal. The panel consisted of three anesthesiologists, three nurse anesthetists, one endocrinologist, one diabetic nurse educator, one administrator, three physician assistants, two certified registered nurse practitioners, one vascular surgeon, one general surgeon, and two orthopedic surgeons. The panelists worked with diabetic elective surgery 
patients on a variety of points in their service of preoperative care. All panelists had more than 10 years of experience in their field.

The following list outlines the proposed CPG:

1. Patient identified as a possible surgical candidate should be screened when identified as high risk if they have Type I diabetes, Type II diabetes, take insulin, take oral hypoglycemics, or have a BMI $\geq 28$ $\mathrm{kg} / \mathrm{m} 3$.

2. For "high risk" patients, $\mathrm{HgbA1C}$ results are to be reviewed if drawn within three months of preadmission center appointment. If not done, $\mathrm{HgbA1C}$ test to be drawn as soon as possible.

3. If patient has $\mathrm{HgbA1C}$ result $<8 \%$, proceed with surgery as planned.

4. If $\mathrm{HgbA1C}$ result is $\geq 8 \%$, surgery is postponed and patient is referred to endocrinology or primary care physician for optimization.

5. Patient receives handouts, a referral to the diabetes education center, and a letter of condition for the endocrinologist or primary care physician outlining the need for diabetes optimization and goal necessary to reschedule surgery.

6. Patient to return to preadmission center after 8 weeks with a $\mathrm{HgbA1C}$ report after optimization if result is $<8 \%$. If level does not meet criteria, optimization will continue until goal level is reached.

\section{Results}

The AGREE II tool (Brouwers et al., 2010) is used internationally to assess the quality of a CPG. The AGREE collaboration established the reliability and validity of the AGREE II tool (AGREE Collaboration, 2003).

- Content validity: 95\% of appraisers found the instrument useful for assessing guidelines.

- Reliability: internal consistency ranged between 0.64 and 0.88 .

- Face validity: results from the first field test indicated that the appraisers found the instrument useful to assess guidelines (95\%) and the user guide helpful (98\%).

Eighteen expert panelists completed a review of the proposed CPG utilizing the AGREE II tool. The final overall score for the quality of the guideline was $96.2 \%$ with all experts stating they recommended the CPG. Fourteen of the expert panelists made the same recommendation to modify the HgbA1C acceptable result from $8.0 \mathrm{mg} / \mathrm{dl}$ to $8.5 \mathrm{mg} / \mathrm{dl}$. Six expert panelists recommended adding a periodic review process to ensure evidence-based efficacy for the CPG.

Based on these recommendations, the CPG was adjusted to include these recommendations. A formative evaluation process will take place three months after implementation. Once again, recommendations and changes can be discussed and addressed. Finally, a summative evaluation process can be conducted again after six more months of implementation. More adjustments can be made if needed. The goal for end results is the reduction of surgery cancellations for diabetic patients having elective surgery. The goal reduction in surgery cancellation rate is set for $<5 \%$. 
The following list outlines the final CPG:

1. Patient identified as a possible surgical candidate should be screened when identified as high risk if they have Type I diabetes, Type II diabetes, take insulin, take oral hypoglycemics, or have a BMI $>28 \mathrm{~kg} / \mathrm{m} 3$.

2. For "high risk" patients, $\mathrm{HgbA1C}$ results are to be reviewed if drawn within three months of preadmission center appointment. If not done, $\mathrm{HgbA1C}$ test to be drawn as soon as possible.

3. If patient has $\mathrm{HgbA1C}$ result $<8.5 \%$ proceed with surgery as planned.

4. If $\mathrm{HgbA1C}$ result is $>8.5 \%$, surgery is postponed, and patient is referred to endocrinology or primary care physician for optimization.

5. Patient receives handouts, a referral to the diabetes education center, and a letter of condition for the endocrinologist or primary care physician outlining the need for diabetes optimization and goal necessary to reschedule surgery.

6. Patient to return to preadmission center after 8 weeks with a $\mathrm{HgbA1C}$ report after optimization if result is $<8.5 \%$. If level does not meet criteria, optimization will continue until goal level is reached.

7. A formative evaluation will be completed at the 3-month mark to include an evaluation process postimplementation of the CPG.

8. A summative evaluation will be completed at the 6-month mark to assess the CPG impact on the rate of unnecessary surgery cancellations.

\section{Strengths and Limitations}

Cancellation of elective surgeries on the day of the procedure precedes unproductive use of operating room time and a waste of resources. Day-of-surgery cancellations also instigate trouble for patients and families. Moreover, day-of-surgery cancellation creates logistic and financial burdens associated with extended hospital stay and repetition of preoperative preparations as well as opportunity costs of lost time and missed income (Kaddoum et al., 2016). Having a clinical practice guideline to reduce unnecessary cancellations related to poorly optimized cases will impact these issues. This clinical practice guideline has been developed for this clinical site but is also applicable to other health care facilities. A significant strength of this project was the support of the stakeholders to agree to be a part of the expert panel. Because a sample from each discipline considered to be a major stakeholder was involved in the critique of the clinical practice guideline, it is expected that adoption of the guideline will be without incident. Buy-in is supported by the results of the survey. Limitations related to the continued success of the clinical practice guideline would be advanced practice nurse, anesthesiology, and surgeon turn-over in practice. It is important for the quality assurance process to stay in effect to ensure continued success.

\section{Recommendations for Future Projects}

A future prospect to disseminate the findings of this CPG implementation would be submitting the clinical practice guideline to other healthcare systems' quality improvement teams for their review. This would allow the clinical practice guideline to be disseminated to other local facilities in the area. As others are reviewing the content locally, the guideline could be disseminated to other similar-sized healthcare facilities throughout the state of Pennsylvania. In addition, this facility would benefit from the development of other CPGs, such as hypertension and cardiac arrhythmias, because these clinical issues frequently occur as reasons for OR cancellations. The CPG could be used as a guideline for the development of future CPGs at this facility. 
Harbaugh \& Whitehead, 2020

\section{References}

AGREE Collaboration (2003). Development and validation of an international appraisal instrument for assessing the quality of clinical practice guidelines: the AGREE project. Quality and Safety in Health Care 12, 18-23 https://doi.org//10.1136/qhc.12.1.18.12571340

American Diabetes Association. (2019). Standards of medical care in diabetes-2019. Diabetes Care: The Journal of Applied Research and Education, 42, Supp. 1, S1-S193. https://doi.org/10.2337/dc19-Sinto1

Brouwers, M., Kho, M. E., Browman, G. P., Cluzeau, F., Feder, G., Fervers, B., Hanna, S., \& Makarski, J. on behalf of the AGREE Next Steps Consortium (2010). AGREE II: Advancing guideline development, reporting and evaluation in healthcare. Canadian Medical Association Journal, 182, E839-842. https//doi.org/10.1503/cmaj.090449

Bunker, K. A. (2006). Leading change: A balancing act. Nurse Leader, 4(1), 43-45. https://doi.org//10.1016/j.MN/2006.01.004

Kaddoum, R., Fadlallah, R., Hitti, E., Jardali, F., \& Eid, G. (2016). Causes of cancellations on the day of surgery at a tertiary teaching hospital. BMC Health Services Research, 16(259), 1-8. https://doi.org/10.1186/s12913-016-1475-6

LaBoone, L. M., McLarney, J. T., \& Reynolds, L. R. (2014). An interdepartmental collaboration to improve preoperative glycemic control. Hospital Practice, 42(5), 83-88. https://doi.org/10.3810/hp.2014.12.1161

Lee, G. A., Wyatt, S., Walker, K. Z., Topliss, D., \& Stoney, R. (2014). A study of pre-operative intervention in patients with diabetes undergoing cardiac surgery. Collegian, 21, 287-293. https://doi.org/10.1016/j.colegn.2013.06.001

Levy, N., \& Dhatariya, K. (2019). Preoperative optimization of the surgical patient with diagnosed and undiagnosed diabetes: A practical review. Anesthesia, 74(Supp. 1), 3-6. https://doi.org/10.1111/anae.14510

Mendez, C. E., Wainaina, N., Walker, R. J., Montagne, W., Livingston, A., Slawski, B., \& Egede, L. E. (2018). Preoperative diabetes optimization program. Clinical Diabetes Journal, 36(1), 68-71. https://doi.org/10.2337/cd17-0088

Minor, D. O. (2018). Implementing a surgical pathway to reduce operating room cancellation rates. [Unpublished doctoral dissertation]. University of South Carolina. https://scholarcommons.sc.edu/etd/4562

Nelson-Brantley, H. V., \& Ford, D. J. (2017). Leading change: a concept analysis. Journal of advanced nursing, 73(4), 834-846. https://doi-org.ezp.waldenulibrary.org/10.1111/jan.13223

Rollins, K. E., Varadhan, K. K., Dhatariya, K., \& Lobo, D. N. (2016). Systematic review of the impact of HbA1c on outcomes following surgery in patients with diabetes mellitus. Clinical Nutrition, 35, 308-316. https://doi.org/10.1016/j.clnu.2015.03.007

Turner, R. M., Ma, Q., Lorig, K., Greenberg, J., \& DeVries, A. R. (2018). Evaluation of a diabetes selfmanagement program: Claims analysis on comorbid illnesses, health care utilization, and cost. Journal of Medical Internet Research, 20(6), 207. https://doi.org/10.2196/jmir.9225

Underwood, P., Hurwitz, S., Chamarthi, B., \& Garg, R. (2014). Preoperative A1C and clinical outcomes in patients with diabetes undergoing major noncardiac surgical procedures. Diabetes Care, 37(3), 611616. https://doi.org/10.2337//dc13-1929

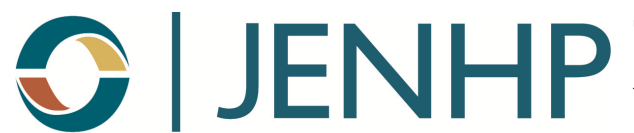

The Journal of Excellence in Nursing and Healthcare

Practice welcomes manuscripts focusing on topics such as national and international workforce issues, quality improvement projects, evidence-based practice initiatives, nursing research studies, interprofessional practice, educational issues, telehealth, improvements in technology, and the impact of social change in society. 